\title{
SiO IN STAR FORMING REGIONS: BARNARD 1 AND ORION KL
}

\author{
SATOSHI YAMAMOTO, HITOMI MIKAMI \\ Department of Astrophysics, Nagoya University, Chikusa, Nagoya 464-01, \\ Japan \\ SHUJI SAITO \\ Institute for Molecular Science, Okazaki 444, Japan
}

\begin{abstract}
Interferometric observations of the SiO $(J=2-1)$ line are carried out toward a low-mass-star forming region, B1, and a massive star forming region, Ori KL. Production mechanisms of $\mathrm{SiO}$ in these regions are discussed.
\end{abstract}

\section{INTRODUCTION}

$\mathrm{SiO}$ is the most fundamental $\mathrm{Si}$ containing interstellar molecule. This molecule has long been observed in the massive-star forming regions like Ori KL and NGC7538 and supernova remnamt IC443 (e.g. Ziurys et al. 1989). Recently, Bachiller et al. (1990) detected the SiO $(J=3-2)$ line toward Barnard 1 (B1). Subsequently SiO has been detected in other low-mass-star forming regions, L1448 (Bachiller et al. 1991) and L1157 (Mikami et al. 1992). These results indicate that $\mathrm{SiO}$ is produced even in less-active star forming regions. In order to understand the formation processes of $\mathrm{SiO}$ in such regions, we studied a distribution of SiO in B1 with Nobeyama Millimeter Array (NMA) (Yamamoto et al. 1992). We also obtained the SiO distribution in Ori KL for comparison.

\section{SiO IN BARNARD 1}

Figure 1 shows the integrated intensity map of the $\mathrm{SiO}(J=2-1)$ line toward B1. A synthesized beam is $8 " \times 10$ ". Distribution of SiO consists of at least two compact clumps. The clump A is possibly apart from the IRAS source, although uncertainty of the IRAS position is large. The blue lobe of the CO $(J=1-0)$ outflow is distributed toward the south-west direction from the IRAS source (Nakayama 1988). However, a red-shifted wing component was not detected in their observations. In contrast, the red-shifted wing component is clearly seen in the SiO emission at the clump $\mathrm{A}$. According to the $\mathrm{H}^{13} \mathrm{CO}^{+}$observations, dense gas exists at the eastern side of the clump A. Therefore it is likely that the red lobe of the outflow collides with the dense gas, and, in such a shocked region, $\mathrm{SiO}$ is efficiently produced through evaporation of dust grains.

In $\mathrm{L} 1157$ the $\mathrm{SiO}$ emission arises from an interacting region between molecular outflow and dense gas. Furthermore the SiO emission is found in molecular bullets in the outflow of L1448. These facts along with our results on B1 suggest that interaction between outflow and dense gas plays a major role in the $\mathrm{SiO}$ production in low-mass-star forming regions.

\section{SiO IN ORION KL}

The distribution of SiO $(J=2-1)$ in Ori KL was first studied by Wright et al. (1983) with the Hat Creek interferometer. We obtained higher resolution ( 3 ”) map to investigate interaction between outflow and ambient gas. Figure 2 shows the distribution of the red-shifted component of $\mathrm{SiO}$ superposed on the $\mathrm{H}_{2} \mathrm{CO}$ $\left(2_{11}-2_{12}\right)$ integrated intensity map (Mangum et al. 1990). The SiO emission 
clearly traces the eastern edge of the $\mathrm{H}_{2} \mathrm{CO}$ distribution, suggesting that the outflow is blowing up the ambient cloud. The $\mathrm{SiO}$ emission seems to represent shocked regions in interface between outflow and ambient gas.

The distribution of $\mathrm{SiO}$ in the central velocity range has an unresolved component associated with IRc2 (Wright et al. 1983; Yamamoto et al. 1993). This indicates that $\mathrm{SiO}$ is produced by radiation heating from IRc2. The high velocity component of $\mathrm{SiO}$ shown in Figure 2 may represent a part of outflowing $\mathrm{SiO}$ from IRc2. However, it seems possible that high velocity $\mathrm{SiO}$ is produced in the shocked region apart from IRc2 through the same mechanism as in the case of low-mass-star forming regions.

The authors are grateful to R. Kawabe, Y. Murata, N. Kaifu, M. Ohishi, and K. Kawaguchi for their valuable discussions.

Bachiller, R., Martín-Pintado, J., and Fuente, A., 1991, A\&A, 243, L21.

Bachiller, R., Menten, K.M., and del Rio-Alvarez, S., 1990, A\&A, 236, 461.

Mangum, J.G. et al., 1990, ApJ, 348, 542.

Mikami, H. et al., 1992, ApJ, 392, L87.

Nakayama, M., 1988, M.S. Thesis, Tohoku Univ.

Wright, M.C.H. et al., ApJ, 267, L41.

Yamamoto, S. et al., 1992, PASJ, 44, 459.

Yamamoto, S., Mikami, H., and Saito, S., 1993, in this proceeding.

Ziurys, L.M., Friberg, P., Irvine, W.M., 1989, ApJ, 343, 201.

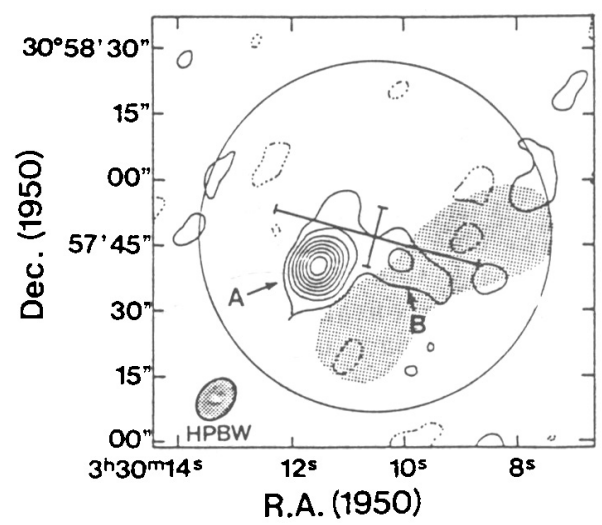

Figure 1: Integrated intensity map of $\mathrm{SiO}(J=2-1)$. The shaded area represents the distribution of the CO blue lobe (Nakayama 1988).

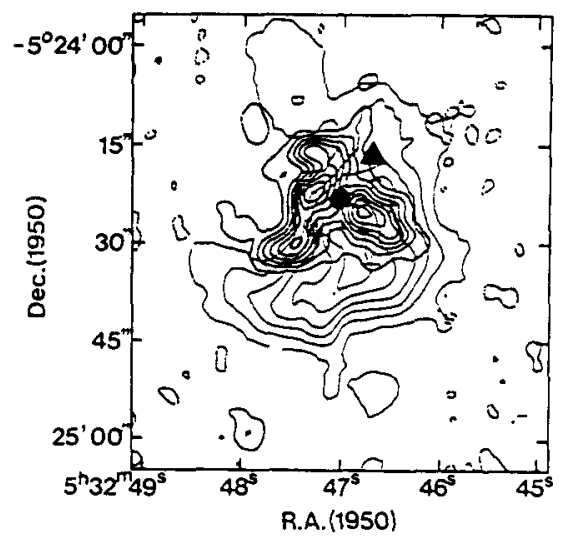

Figure 2: Map of the red-shifted component $\left(26<v<30 \mathrm{~km} \mathrm{~s}^{-1}\right)$ of $\mathrm{SiO}(J=2-1)$ (thick contours) superposed on a total integrated intensity map of $\mathrm{H}_{2} \mathrm{CO}$ (Mangum et al. 1990; thin contours). 